\title{
MUTUAL COUPLING ANALYSIS OF A DUAL CIRCULARLY POLARISED CONTRAWOUND QUADRIFILAR HELIX ANTENNA (CQHA) IN LAND MOBILE SATELLITE (LMS) MIMO SYSTEMS
}

\author{
M. F. Mansor, T. W. C. Brown and B. G. Evans \\ Centre for Communication Systems Research, University of Surrey, UK. \\ \{M. Mansor, T.Brown, B.Evans\}@surrey.ac.uk
}

Keywords: Mutual coupling, CQHA, LMS MIMO.

\begin{abstract}
Analysis of the mutual coupling and its effect on the CQHA performance in LMS MIMO system are investigated and presented in this paper. Due to the complexity of the CQHA structure, the mutual coupling effects were mainly studied using electromagnetic simulation. The effect of the separation spacing between inner and outer QHA on the mutual coupling was investigated by comparing the element isolation of CQHAs with varying outer QHA radius and fixed inner QHA radius. It can be concluded that a minimum separation of $0.1 \lambda$ is required for the isolation to be better than $15 \mathrm{~dB}$. The distortion of the co-polar and cross-polar radiation patterns of the CQHA due to the mutual coupling are also studied in this paper. Finally, the effect of the mutual coupling on the mean effective gain and polarisation correlation of CQHAs with varying separation spacing between inner and outer QHA were examined and its results showed that the MEG values are very much affected by mutual coupling while the polarisation and angular correlation are largely unaffected.
\end{abstract}

\section{Introduction}

MIMO techniques have become one of the most important technologies in the next generation wireless communication system for its enormous spectral efficiency without additional bandwidth and power. Even so, its implementation in land mobile satellite (LMS) systems is more difficult due to the differences in propagation channel characteristics which render the spatial based MIMO technique impractical. However, by implementing polarisation multiplexing (polarisation based MIMO technique), land mobile satellite systems can also benefit from the capacity increase of MIMO systems [5]. Therefore, a compact dual circularly polarised antenna with upper hemispherical radiation pattern is needed for LMS receiver terminal. The dual circularly polarised Contrawound Quadrifilar Helix Antenna (CQHA) has been introduced in [6] as one of the promising candidates for Land Mobile Satellite MIMO receiver terminal due to its dual orthogonal polarisation capability while retaining the desirable wide beam circular polarised pattern.
Capacity of the MIMO systems is mainly dependent on the signal correlation at the transmitter and the receiver, which can be categorised into two categories; propagation induced correlation and antenna induced correlation [2]. The signal correlation needs to be low in order for the MIMO system to fully operate at its optimum capacity. Effect of the mutual coupling on the capacity of MIMO system is then related to the impact of mutual coupling on the signal correlation and the received signal-to-noise ratio (SNR) [4]. It is known that mutual coupling between closely spaced radiating elements can distorts the element radiation pattern and also reduces the radiation efficiency due to impedance mismatch of the element [7]. The distortion of the element pattern can have different impacts on the MIMO system depending on the propagation channel correlation. In a poor scattering environment with small angular spread and correlated subchannels, distortion of antennas radiation patterns function as decorrelator where the dissimilar patterns create pattern diversity which reduce the overall signal correlation [8]. As for a rich multipath environment with fully uncorrelated subchannels, mutual coupling can increases the signal correlation and reduces the theoretical capacity of the MIMO system [3].

In this paper, the effect of mutual coupling on the dual circularly polarised CQHA radiation patterns is presented. Then, more studies on its effect on the mean effective gain and polarisation correlation of the CQHA were conducted and its results and discussions are shown in Chapter 5. The conclusion of the studies is given at the end of the paper.

\section{CQHA Design and Parametric Study}

The design of CQHA is based on the combination of two QHAs with opposite element winding direction to provide dual circular polarisation capability. In order to reduce the size of the antenna, one QHA is positioned inside of the other. A major disadvantage of this structure is strong mutual coupling between antenna elements due to its close proximity. However, such mutual coupling effect can be reduced by increasing the spacing between inner and outer QHA. This can be achieved by increasing the radius of the outer QHA 
while keeping the radius of the inner QHA fixed. This method, however, is limited by the maximum radius achievable by element length of the QHA and its effect on the radiation pattern of the outer QHA. Configuration of the proposed CQHA and its element/port numbering are shown in Fig. 1. In order to investigate the effect of the mutual coupling on the CQHA performance, a parametric study has been conducted by electromagnetic simulation using CST Microwave Studio where spacing between inner and outer QHA is varied from $10 \mathrm{~mm}$ to $15 \mathrm{~mm}$ and its effect on the CQHA performances are studied.

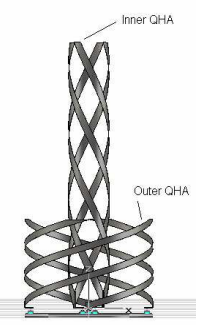

(a)

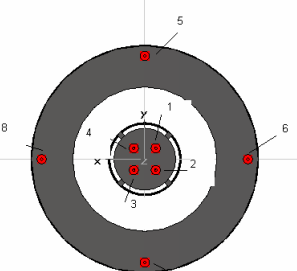

(b)
Fig 1. a) Configuration of the CQHA and b) Its elements/ports numbering.

Table 1. The physical parameters of the simulated CQHAs with varying separation spacing between inner and outer QHA.

\begin{tabular}{l|c|c|c|c|c|c|c}
\hline Parameter & $\begin{array}{l}\text { Inner } \\
\text { QHA }\end{array}$ & \multicolumn{7}{|c}{ Outer QHA } \\
\hline Radius / mm & 7 & 17 & 18 & 19 & 20 & 21 & 22 \\
\hline Helical turn & 1 & 0.75 & 0.75 & 0.75 & 0.75 & 0.75 & 0.75 \\
\hline $\begin{array}{l}\text { Element } \\
\text { length / } \\
\text { wavelength }\end{array}$ & 0.75 & 0.75 & 0.75 & 0.75 & 0.75 & 0.75 & 0.75 \\
\hline $\begin{array}{l}\text { Axial length / } \\
\text { mm }\end{array}$ & 90 & 70 & 64 & 58 & 50 & 42 & 30 \\
\hline Spacing /mm & - & 10 & 11 & 12 & 13 & 14 & 15 \\
\hline
\end{tabular}

\section{Separation Spacing Effect on the Mutual Coupling of the CQHA}

It is well known that mutual coupling between radiating elements are inversely proportional to the distance between the elements. Mutual coupling can be represented by the element isolation from scattering parameter simulation or measurement. In this study, the effect of varying the radius of the outer QHA on the mutual coupling, which effectively increase the spacing between inner and outer QHA, is examined.

Simulated isolation between element 1 on the inner QHA and element 5 on the outer QHA are chosen as the indicator for the mutual coupling between inner and outer QHA as shown in Fig. 2. Based on the results, it clearly shows that in order for the element isolation to be better that $15 \mathrm{~dB}$, the spacing between inner and outer QHA has to be $15 \mathrm{~mm}$ or $0.1 \lambda$ at $2 \mathrm{GHz}$ frequency.

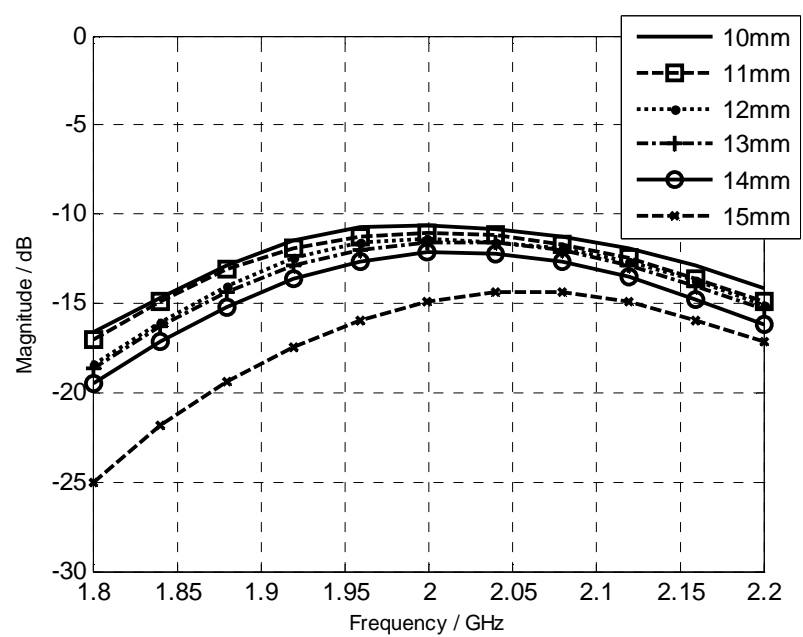

Fig. 2 Isolation between element 1 on the inner QHA and element 5 on the outer QHA for CQHAs with separation spacing between $10 \mathrm{~mm}$ to $15 \mathrm{~mm}$.

\section{Mutual Coupling Effect on Radiation Pattern}

One of the main advantages of QHA is its wide circularly polarised beam and it is crucial for the CQHA to exhibit the same radiation pattern. It has been stated that mutual coupling can distorts element radiation pattern depending on the spacing of the elements. Therefore, the effect of mutual coupling on the CQHA pattern with varied spacing between inner and outer QHA need to be characterised. This can be done by evaluating the radiation pattern of the radiating QHA when the radiating QHA is excited by unit voltage source while the other QHA is terminated with its generator impedance. The resultant co-polar and cross polar radiation patterns from this method are the pattern of the antenna in presence of mutual coupling due to its proximity with another antenna.

In this study, the simulated co-polar and cross polar elevation pattern of the inner QHA and outer QHA of CQHAs with separation spacing of $10 \mathrm{~mm}$ and $15 \mathrm{~mm}$ are compared with isolated inner QHA and outer QHA (radius of 22mm) as shown in Fig. 3 and Fig. 4. Based on the results, it is clearly shows that mutual coupling has detrimental effect on the copolar and cross-polar pattern of inner and outer QHA. The copolar gain of the inner QHA is reduced by nearly $7 \mathrm{~dB}$ at the zenith when inner QHA is positioned inside an outer QHA with radius of $17 \mathrm{~mm}$. By increasing the radius of outer QHA up to $22 \mathrm{~mm}$, the co-polar gain of inner QHA has improved by almost $4 \mathrm{~dB}$ at the zenith. The co-polar elevation patterns for outer QHA are also clearly improved with increasing spacing between the two antennas. Increase in gain are evident where 4 to $5 \mathrm{~dB}$ improvement of the pattern from $30^{\circ}$ (from horizontal plane) to the zenith. However, due to the increased radius of the outer QHA, its co-polar elevation pattern becomes more directional as its $3 \mathrm{~dB}$ beamwidth decreases from $186^{\circ}$ to $101^{\circ}$. Meanwhile, the cross-polar radiation pattern for inner and outer QHA are significantly increased by the effect of mutual coupling which can be shown by comparing the cross-polar pattern of isolated inner and outer 
QHA and the CQHA with separation spacing of $10 \mathrm{~mm}$ and $15 \mathrm{~mm}$. These effects can be explained by considering that the induced current at the terminated QHA is in phase with the current at the excited QHA which then causes the terminated QHA to radiate the orthogonal polarisation but at the opposite direction due to feeding phase direction.

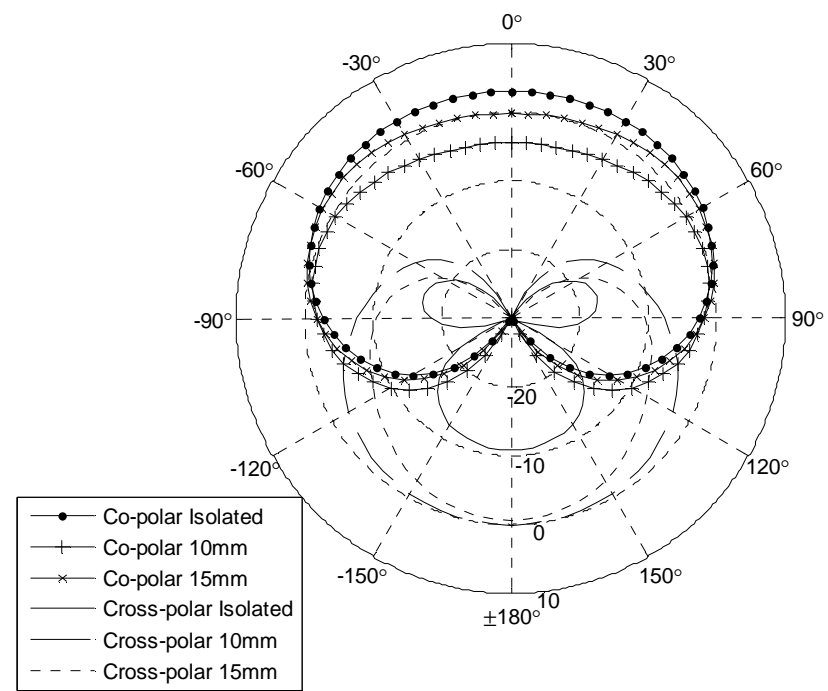

Fig. 3. The co-polar and cross-polar elevation radiation pattern for isolated inner QHA, inner QHAs of CQHAs with separation spacing of $10 \mathrm{~mm}$ and $15 \mathrm{~mm}$ between inner and outer QHA (dBiC)

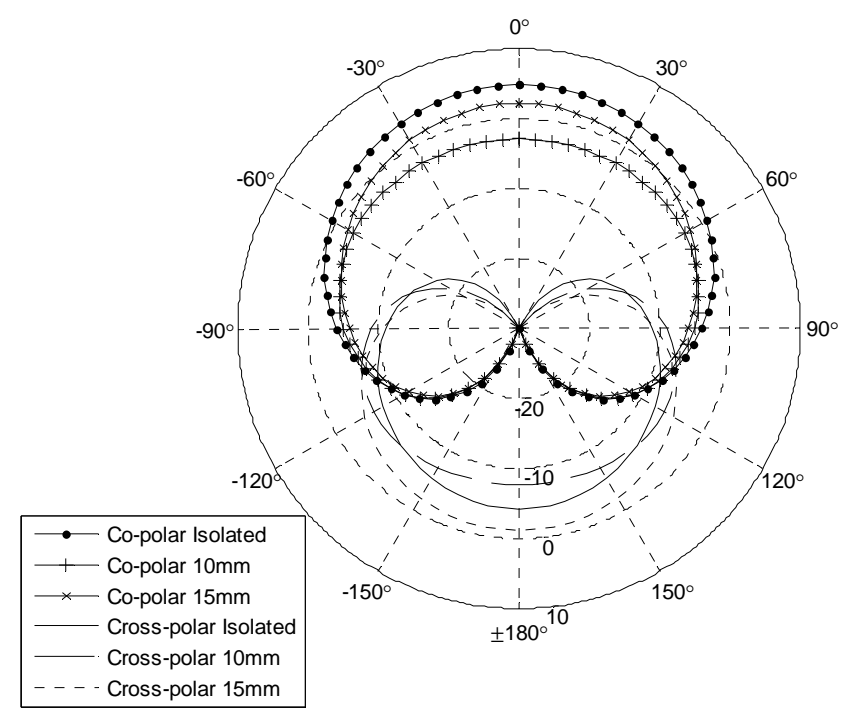

Fig. 4. The co-polar and cross-polar elevation radiation pattern for isolated outer QHA, outer QHAs of CQHAs with separation spacing of $10 \mathrm{~mm}$ and $15 \mathrm{~mm}$ between inner and outer QHA (dBiC)

\section{Mutual Coupling Effect on Mean Effective Gain and Correlation}

\subsection{Mean Effective Gain}

The analysis of the mean effective gain (MEG) of the CQHAs with separation spacing from $10 \mathrm{~mm}$ to $15 \mathrm{~mm}$ was done using closed form formulation by Taga [9] as listed below.

$$
M E G=\int_{0}^{2 \pi} \int_{0}^{\pi}\left(\frac{X P R}{1+X P R} G_{\theta}(\theta, \phi) P_{\theta}(\theta, \phi)+\frac{1}{1+X P R} G_{\phi}(\theta, \phi) P_{\phi}(\theta, \phi)\right) \sin \theta d \theta d \phi
$$

where XPR is the cross polarisation power ratio, $G_{\theta, \phi}(\theta, \phi)$ are the theta/phi components of the antenna power gain pattern and $P_{\theta, \phi}(\theta, \phi)$ are the theta/phi components of the angular density function of the Angle of Arrival (AOA) of the incident waves. The azimuth distribution of the AOA for both polarisations is uniform due to randomness in terminal orientation and scatterers distribution while the elevation distribution is Gaussian with varying mean and a fixed standard deviation. In order to investigate the effect of mutual coupling on the MEG of the inner and outer QHA, the MEG is calculated for a fixed XPR, which in this case is $0 \mathrm{~dB}$. Results of the MEG analysis of the CQHAs with separation spacing from $10 \mathrm{~mm}$ to $15 \mathrm{~mm}$ are shown in Fig. 5 for inner QHA and Fig. 6 for outer QHA.

For both QHAs, the MEG has the lowest values when the separation spacing is $10 \mathrm{~mm}$ and increases as the separation is increased from $10 \mathrm{~mm}$ to $15 \mathrm{~mm}$. At elevation angle of $60^{\circ}$ (from horizontal plane), the difference of MEG value for inner and outer QHA is more than $3 \mathrm{~dB}$. This effect can be easily explained as the co-polar gain pattern for both QHAs is improved as the separation is getting larger which then affects the MEG value of the antennas. When the separation spacing is $15 \mathrm{~mm}$, the maximum value of the MEG of the inner QHA is obtained when the mean of elevation distribution is at $40^{\circ}$ from the horizontal plane while for the outer QHA, its Meg value reaches its maximum at $60^{\circ}$.

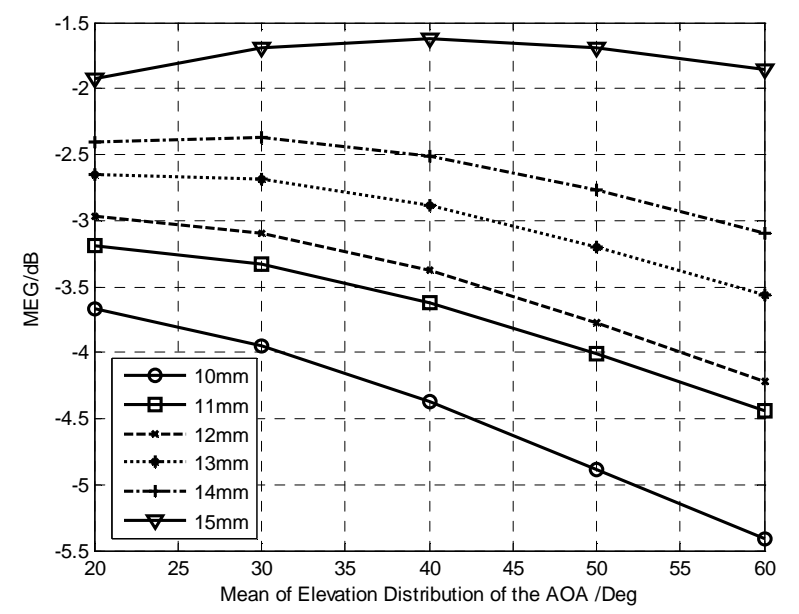

Fig. 5. MEG value for inner QHA of CQHAs with separation spacing of $10 \mathrm{~mm}$ to $15 \mathrm{~mm}$ between inner and outer QHA. 


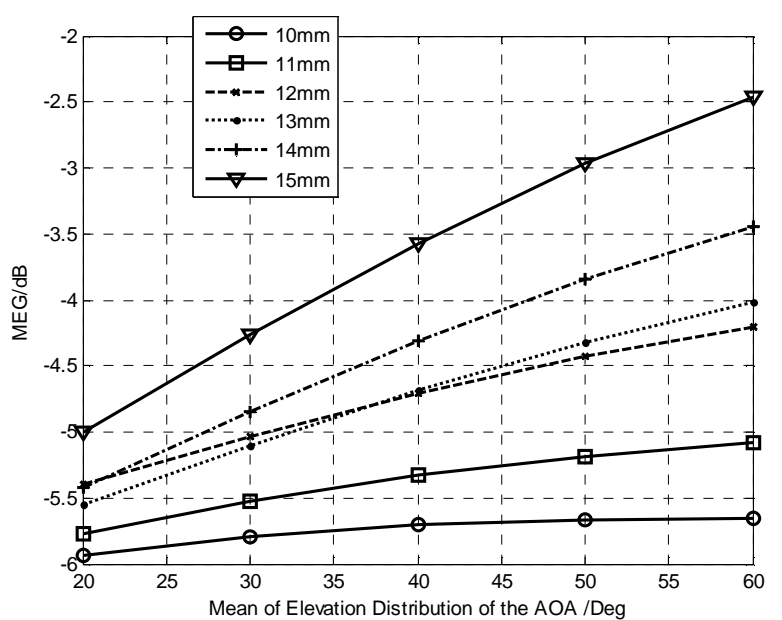

Fig. 5. MEG value for inner QHA of CQHAs with separation spacing of $10 \mathrm{~mm}$ to $15 \mathrm{~mm}$ between inner and outer QHA.

\subsection{Correlation}

Realistic capacity increase of a polarisation-based MIMO system depends on the polarisation correlation of the dual orthogonal polarised antennas used in the system, apart from depolarisation effect of the propagation channel. Calculation of polarisation correlation of the CQHAs with separation spacing from $10 \mathrm{~mm}$ to $15 \mathrm{~mm}$ can be done by using formulation in [6] which gave closed form equation for circular polarisation correlation coefficient.

In evaluating polarisation correlation of the CQHAs, the same channel parameters in MEG analysis have been used for the value of XPR and AOA. The complex polarisation correlation coefficients for the CQHAs with separation spacing from $10 \mathrm{~mm}$ to $15 \mathrm{~mm}$ between inner and outer QHA are shown in Table 2. The results indicate that the polarisation correlation of the CQHA is very low $(<0.1)$ even at the separation spacing of $10 \mathrm{~mm}$ between inner and outer QHA. In fact, the polarisation correlation of the CQHA is lower at separation spacing of $10 \mathrm{~mm}$ compared to $15 \mathrm{~mm}$. It is stated in [1] that the polarisation correlation is inherent in the angular correlation and it can be expected that the angular correlation of the CQHA will be about the same value. Table 3 shows that complex angular correlation coefficient of the CQHA for separation spacing between $10 \mathrm{~mm}$ to $15 \mathrm{~mm}$ for inner and outer QHA.

It was expected that as the separation spacing of the CQHA is reduced, the polarisation and angular correlation of the CQHA are increased. However, based on the correlation analysis, the polarisation correlation coefficient is largely unaffected by the mutual coupling of the CQHA elements. One explanation for this effect is although the inner and outer QHA are closely spaced and exhibit strong mutual coupling, both antennas are still radiate almost perfectly orthogonal circular polarisations at the upper hemispherical plane which cause the polarisation and angular correlation coefficient to be very low. But of course, such close spacing between QHAs reduces the radiation efficiency and gain pattern of the antennas as shown in the MEG analysis earlier.

Table 2. The complex polarisation correlation coefficient of the CQHAs with separation spacing from $10 \mathrm{~mm}$ to $15 \mathrm{~mm}$ between inner and outer QHA.

\begin{tabular}{c|c|c}
\hline \multirow{2}{*}{$\begin{array}{l}\text { Separation } \\
\text { distance }(\mathbf{m m})\end{array}$} & \multicolumn{2}{|c}{ Mean of the Elevation Distribution } \\
\cline { 2 - 3 } $\mathbf{2 0}$ & $\mathbf{6 0}$ \\
\hline $\mathbf{1 0}$ & $0.0054+0.0049 \mathrm{i}$ & $0.0059+0.0082 \mathrm{i}$ \\
$\mathbf{1 1}$ & $0.0045+0.0041 \mathrm{i}$ & $0.0052+0.0065 \mathrm{i}$ \\
$\mathbf{1 2}$ & $0.0044+0.0045 \mathrm{i}$ & $0.0050+0.0069 \mathrm{i}$ \\
$\mathbf{1 3}$ & $0.0051+0.0046 \mathrm{i}$ & $0.0061+0.0064 \mathrm{i}$ \\
$\mathbf{1 4}$ & $0.0044+0.0052 \mathrm{i}$ & $0.0048+0.0069 \mathrm{i}$ \\
$\mathbf{1 5}$ & $0.0130+0.0067 \mathrm{i}$ & $0.0163+0.0066 \mathrm{i}$ \\
\hline
\end{tabular}

Table 3. The complex angular correlation coefficient of the CQHAs with separation spacing from $10 \mathrm{~mm}$ to $15 \mathrm{~mm}$ between inner and outer QHA.

\begin{tabular}{c|c|c}
\hline $\begin{array}{l}\text { Separation } \\
\text { distance }(\mathbf{m m})\end{array}$ & \multicolumn{2}{|c}{ Mean of the Elevation Distribution } \\
\cline { 2 - 3 } $\mathbf{2 0}$ & $\mathbf{6 0}$ \\
\hline $\mathbf{1 0}$ & $0.0054+0.0048 \mathrm{i}$ & $0.0059+0.0079 \mathrm{i}$ \\
$\mathbf{1 1}$ & $0.0045+0.0041 \mathrm{i}$ & $0.0052+0.0063 \mathrm{i}$ \\
$\mathbf{1 2}$ & $0.0044+0.0046 \mathrm{i}$ & $0.0049+0.0068 \mathrm{i}$ \\
$\mathbf{1 3}$ & $0.0052+0.0047 \mathrm{i}$ & $0.0060+0.0063 \mathrm{i}$ \\
$\mathbf{1 4}$ & $0.0044+0.0054 \mathrm{i}$ & $0.0048+0.0068 \mathrm{i}$ \\
$\mathbf{1 5}$ & $0.0137+0.0069 \mathrm{i}$ & $0.0162+0.0065 \mathrm{i}$ \\
\hline
\end{tabular}

\section{Conclusion}

A dual circularly polarised CQHA has been investigated in terms of its mutual coupling characteristics and its effect on its performance in polarisation-based MIMO system. By increasing the radius of the outer QHA, the mutual coupling between inner and outer QHA can be reduced to $-15 \mathrm{~dB}$. As expected, the mutual coupling distorts the CQHA radiation patterns and reduces the co-polar pattern gain and increases the cross-polar pattern gain of both antennas. Effect of the mutual coupling on the CQHA performance in MIMO system were investigated in terms of mean effective gain and polarisation correlation of the CQHA. The MEG of both QHAs is clearly affected by the mutual coupling as the MEG values are decreased as the separation spacing is reduced between inner and outer QHA. However, as for the polarisation correlation, it is largely unaffected by mutual coupling due to the fact that the antennas are still radiate almost perfect orthogonal polarisations even at close spacing.

\section{Acknowledgements}

The first author would like to thank Ministry of Higher Education, Malaysia for sponsoring his $\mathrm{PhD}$ study at the University of Surrey, UK. 


\section{References}

[1] T. W. C. Brown, S. R. Saunders and B. G. Evans, "Analysis of mobile terminal diversity antennas," IEE Proc.-Microw. Antennas Propag., vol. 152, no. 1, February 2005.

[2] D. W. Browne, "Exploiting the spatial structure of radio communication channels," Ph.D. Thesis, University of California at Los Angeles, June 2007.

[3] P. N. Flecther, M. Dean and A. R. Nix, "Mutual coupling in multielement array antennas and its influence on MIMO channel capacity," IEE Electron. Lett., vol. 39, no. 4, pp. 342-344, Feb 2003.

[4] R. Janaswamy, "Effect of element mutual coupling on the capacity of fixed length linear arrays," IEEE Antennas Wireless Propag. Lett., vol. 1, pp. 157-160, 2002.

[5] P. R. King and S. Stavrou, "Capacity improvements for a land mobile single satellite MIMO System," IEEE Antenna Wireless Propag. Lett., vol. 5, no. 1, pp. 98-100, 2006.

[6] M. F. Mansor, T. W. C. Brown and B. G. Evans, “A dual circularly polarised contrwound quadrifilar helix antenna for land mobile satellite MIMO system," accepted for EuCAP 2009, 23-27 March 2009.

[7] M. K. Ozdemir, H. Arslan and E. Arvas, "Mutual coupling effect in multi-antenna wireless communication systems," in Proc. IEEE Globecomm 2003, vol. 2. pp. 829-833, 1-5 June 2003.

[8] T. Svantesson and A. Ranheim, "Mutual coupling effects on the capacity of multielement antenna systems," in Proc. IEEE Int. Conf. Acoust., Speech, and Signal Processing, vol. 4, Salt Lake City, UT, May 2001, pp. 2485-2488.

[9] T. Taga, "Analysis of mean effective gain of mobile antennas in land mobile radio environments," IEEE Trans. Veh. Technol., vol. 39, no. 2, pp. 117-131, May 1990. 